\title{
Phase Transition Diagnostic in Iron Telluride by Nonlinear Optical Experiments
}

\author{
I.V. KityK ${ }^{a}$, R. Viennois ${ }^{b, c}$ And K. PluCinski ${ }^{d}$ \\ ${ }^{a}$ Electrical Engineering Dept., Czestochowa University of Technology, Armii Krajowej 17, Czestochowa, Poland \\ ${ }^{b}$ Institut Charles Gerhardt, University Montpellier 2 and CNRS, Pl. E. Bataillon, Montpellier, France \\ ${ }^{c}$ Condensed Matter Physics Department, University of Geneva, 21 Quai E. Ansermet, Geneva, Switzerland \\ ${ }^{d}$ Electronic Department, Military University of Technology, Kaliskiego 2, 00-908, Warsaw, Poland
}

We have applied non-linear optical method (second harmonic generation (SHG) and third harmonic generation (THG)) to monitoring the phase transformation in the iron telluride compounds. The experiment was performed in the reflected geometry in which Temperature dependences of the SHG and THG have unambiguously shown that both methods are sensitive to the occurrence of the anomalies in the SHG and THG features near the phase transitions. However, the temperature positions of maxima are different, which may reflect a fact that in these two techniques the different multi-excited states interact differently with the critical bosons near the phase transitions. The role of the surface is crucial here because the surfaces are very sensitive to the presence of oxygen.

PACS: $42.65 . \mathrm{Ky}$

\section{Introduction}

Since 2008, with discovery of superconductivity in iron pnictogenides, research on superconductivity came back to light and scientist were going into new research directions on intermetallics superconducting compounds [1]. Among these iron based superconducting families, one has recently attracted the attention because of its simpler crystal structure, the iron chalcogenides $[1,2]$. The first reason is that these compounds with 2 formula-unit $\mathrm{Fe}_{1+x} \mathrm{Te}_{1-y} \mathrm{Se}_{y}$ per unit-cell are composed of only one type of layer and therefore there is no charge transfer in this superconducting layer. We also must remark that interstitial sites are partly filled by iron atoms between these layers and we have shown previously that this was the reason of inconsistencies found in the literatures in the study of superconducting and magnetic phase diagram of the iron chalcogenide family. Indeed, when the amount of iron, $x$, is too high $(x \geq 0.05)$, the superconductivity is suppressed for Se content, $y<0.45$. For all iron content, no superconductivity is observed when $y<0.2$ [3]. Another interesting point is that the magnetic ordering of the $\mathrm{Fe}_{1+x}$ Te parent compound is different than in the parent compounds of the other family of Fe-based superconductors and that the nature of the magnetic ground state is dependent on the concentration of iron in the interstitial site $[2,3]$. We believe that it is interesting to study these $\mathrm{Fe}_{1+x} \mathrm{Te}$ compounds by nonlinear optical experiments for the following reasons:

- Non-linear optical experiments are good tools for probing phase transitions and their nature [4-6].

- Time resolved experiments in general and among them, time resolved non-linear optical experiments, are efficient tools for probing interaction between electrons and holes or charge carriers as well as bosons and for determining the nature of these last ones, the dynamics of these interactions, notably during magnetic, magneto-structural, superconducting transitions and so on $[4,6-8]$.

The above reasons were the main ones motivating us to make the present study on non-linear optical properties of iron telluride.

However, before making time-resolved experiments, as a first step, we need to obtain reliable and reproducible results for non-linear optical response experiments and to solve possible surface problems that perclude reliable time-resolved experiments.

\section{Experiment}

The samples studied are single-crystalline $\mathrm{Fe}_{1+x} \mathrm{Te}$ with two different iron contents $(x=1.08$ and 1.13) grown by modified Bridgman method. Details of synthesis and sample characterization can be found in [3]. Magnetic properties were measured using a MPMS Squid magnetometer from Quantum Design.

The experimental set-up for non-linear optical (NLO) experiment is shown in Fig. 1.

In the Fig. 1 is depicted a principal set-up for measurements of the nonlinear optical setup. The source light from $10 \mathrm{~ns}$ with frequency repetition about $10 \mathrm{~Hz}$ has passed through the shutter on the sample put in the cryostat. The crystat allows to vary both temperature as well to put the sample in the atmosphere of argon immediately after cleaving. Due to relatively low sizes of the samples (about $0.5 \times 0.8 \mathrm{~mm}$ ) the studies were done by averaging over many pulses. The reflected light was analyzed by the filter which was transparent for $532 \mathrm{~nm}$ in the case of the SHG and $355 \mathrm{~nm}$ for the case of the 


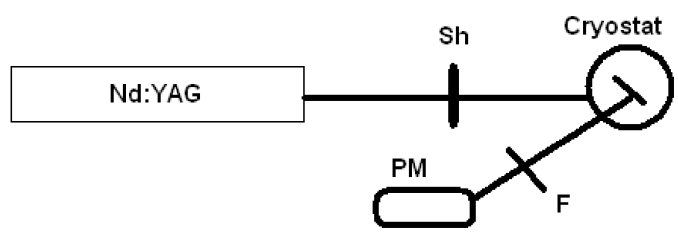

Fig. 1. Principal set-up for measurements of the second harmonic generation (SHG) and third harmonic generation(THG).

THG. The fast response photomultiplier has analyzed the reflected light and performed comparison with the reference samples of PbTe. Very crucial for such kinds of NLO experiments are the surfaces because the useful signal is obtained from the layers of about $200 \mathrm{~nm}$ thickness. Because the titled (?) samples are very sensitive to oxygen, we have done experiments after cleaning of the surfaces by the same laser pulses with higher power and we have detected better sensitivity to the variation of temperature near the phase transitions.

The Nd:YAG laser beam with a power density about 1 $\mathrm{GW} / \mathrm{cm}^{2}$ during 1-2 minute performed the preliminary cleaning of the samples which led to enhanced reflected light intensity. After that measurements were done. The thermo stabilization was performed within the $0.2 \mathrm{~K}$ in the regime of cooling and heating.

\section{Results and discussion}

To understand the sensitivity of the nonlinear optical constants to the observed phase transitions we present below some phenomenological description of the NLO responses.

Polarization of the studied sample can be defined as following $[4,5,9]$ :

$$
\begin{aligned}
& \boldsymbol{P}_{i}=\boldsymbol{P}_{i}^{\mathrm{L}}+\boldsymbol{P}_{i}^{\mathrm{NL}}=\alpha_{i j} E_{j}^{(\omega)} \\
& \quad+\beta_{i j k} E_{j}^{(\omega)} E_{k}^{(\omega)}+\gamma_{i j k l} E_{j}^{(\omega)} E_{k}^{(\omega)} E_{l}^{(\omega)}
\end{aligned}
$$

where $\boldsymbol{P}_{i}^{\mathrm{L}}$ and $\boldsymbol{P}_{i}^{\mathrm{NL}}$ are, the linear and non linear contributions to the polarization, respectively. The second term in the right-hand side of the above equation corresponds to the first order non-linear contribution described by third rank polar tensor requiring acentric charge density distribution and therefore to the secondharmonic generation ( $\mathrm{SHG}$ ), while the third term is assigned to the fourth order tensor component determined the third order nonlinear optical response. The real part of this fourth order tensor corresponds to the third harmonic generation (THG) that will be studied also in the present work for the iron telluride. The higher sensitivity of the NLO susceptibilities with respect to the usual linear optical effects is based on the effective interaction of the higher virtual excited levels (responsible for the NLO) with the soft boson modes which possess higher density near the critical points like superconducting phase transitions. In the theory of the phase transitions, such interactions are usually named soft phonon modes and they are determined by condensation of the same phonon modes dues to the relatively strong electron-phonon anharmonic interactions described by the third rank polar tensors [10]. As we have discussed in a previous paper [9], this soft bosons mode can also be paramagnons coming from damped spin collective excitations corresponding to spin fluctuations that are increased when approaching magnetic phase transitions. Situation is a bit different for the THG, where the principal role is played by the fourth rank polar tensor which does not require any noncentrosymmetry. Generally this coefficient is weaker than the SHG, however due to the excitation of the higher number of photoexcited modes its contribution may be comparable with the SHG and partly complimentary.

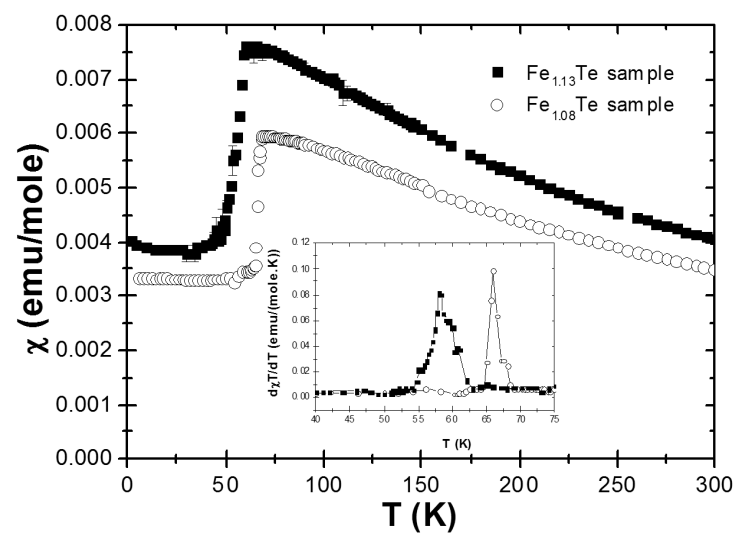

Fig. 2. Thermal variation of the magnetic susceptibility $\chi$, of the two $\mathrm{Fe}_{1+x}$ Te samples with two different $1+x$ iron content. Inset: Thermal variation of $d \chi T / \mathrm{d} T$ for both samples.

Following Fig. 2, one can see that the presence of a strong change in the magnetic susceptibility for both samples and this corresponds to the magneto-structural transition observed in these compounds by using neutron diffraction experiments $[2,11]$. Note that we find that the transition is somewhere broader and at lower temperature for the sample containing the larger amount of iron in interstitial position and this agrees with observation by other groups [2, 11]. For this last sample, the magnetic susceptibility is following Curie-Weiss behavior above $145 \mathrm{~K}$ and we find a Curie-Weiss temperature of about $150 \mathrm{~K}$ and an effective moment $\mu_{\mathrm{eff}}=3.81, \mu_{\mathrm{B}}$ larger than in the first sample. In the inset of the Fig. 2, we show the plot of $d \chi T / \mathrm{d} T$ for highlighting the phase transition and determining the transition temperature. The sample with higher Fe content has a magnetic transition at about $57 \mathrm{~K}, 10 \mathrm{~K}$ lower than the sample with lower iron content.

We have done several different run of NLO experiments on samples coming from the same two batches for which we have reported magnetic properties above. We were able to see THG signal only for the $\mathrm{Fe}_{1.08} \mathrm{Te}$ compound and found an increase of the signal close to the magneto- 


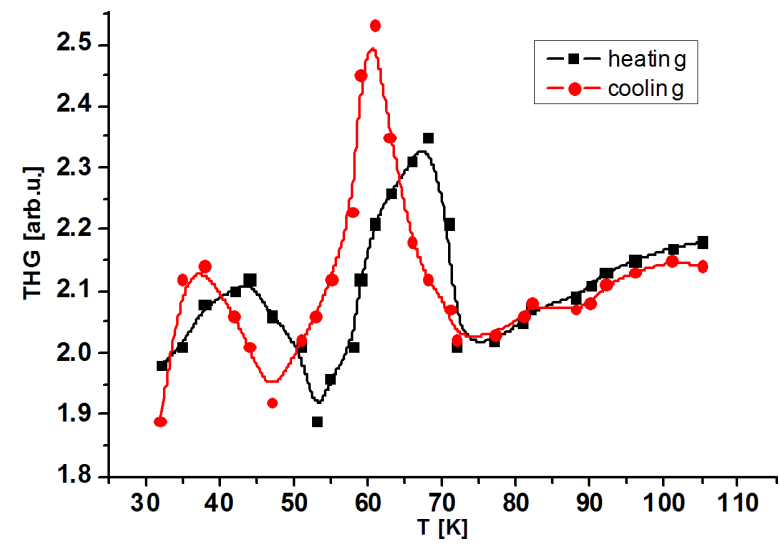

Fig. 3. Thermal variation of the third-harmonic generation signal for the $\mathrm{Fe}_{1.08} \mathrm{Te}$ sample.

structural transition temperature, showing that this technique is able to detect such a kind of transition when sample surface is relatively clean and its roughness allows to detect the mentioned hyperfine interactions. We also see some additional THG anomaly at about $40 \mathrm{~K}$. We can suggest that this could be related to change of electronic structure of the compound when going below the magneto-structural transition due to enhanced interactions of the electronic susbsytem with the critical bosons near the phase transofrmation. The observation of the temperature hysteresis in the THG signal can be related to the first order type of this transition. Concerning SHG experiments, we were able to see some SHG signals in both samples with signal increasing significantly below $80 \mathrm{~K}$ and reaching a maximum between 60 and 70 $\mathrm{K}$ (excepted for the second $\mathrm{Fe}_{1.08} \mathrm{Te}$ sample studied for which only shoulder close to $70 \mathrm{~K}$ is found) and then the SHG signal decreased below about 40-50 K (excepted for the second $\mathrm{Fe}_{1.08} \mathrm{Te}$ sample studied for which the SHG signal is maximal around $40 \mathrm{~K}$ and decreases only below $20-30 \mathrm{~K}$ ). It is interesting to see that we have observed anomalies in thermal variation of SHG signal at the same temperature range, $\mathrm{i}$. e. around $40 \mathrm{~K}$, than for the second peak found in THG signal intensity and that points a probable common origin. But at the present stage of our investigation, even if we are not able to give a clear interpretation of that anomaly, we can remark that the temperature position of this anomaly is close to the minimum observed in the thermopower in $\mathrm{Fe}_{1.08} \mathrm{Te}$ by Pallechi et al [12]. This points the possibility that these anomalies at $40 \mathrm{~K}$ could be due to spin waves in the magnetic ground state. However, there is still no clear interpretation of the observed thermal variation of the thermopower beside the idea that this must be due to magnons. The observation of some SHG signal above the magneto-structural transition temperature can be explained quite similarly to the observation of such kind of signal in the $\mathrm{LaFe}_{4} \mathrm{Sb}_{12}$, i. e. by the increased spin fluctuations [9] that are present just above the transition temperature $\mathrm{T}_{\mathrm{SDW}}$ in the present case. The latter due to enhancement of the higher order electron-boson interactions will stimulate the appearance of the SHG. This is corroborated by the observation of deviation from CurieWeiss between $\mathrm{T}_{\mathrm{SDW}}$ and about $2 \mathrm{~T}_{\mathrm{SDW}}$ that is due to these spin fluctuations.

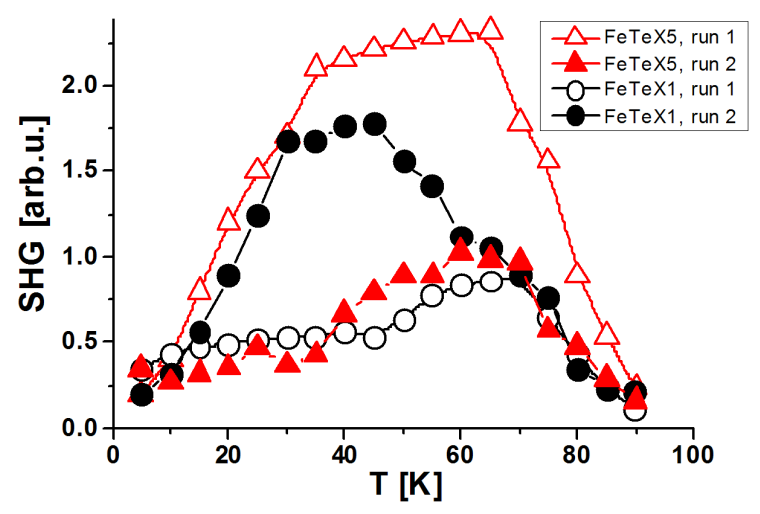

Fig. 4. Thermal variation of second-harmonic generation signal for the two $\mathrm{Fe}_{1+x} \mathrm{Te}$ samples (two different experimental runs).

\section{Conclusions}

We have shown a principal possibity to monitor phase transitions in the iron chalcogenide superconductors by first and second order NLO effects. In particularly, we have established that the THG signal is observed only for the $\mathrm{Fe}_{1.08} \mathrm{Te}$ compound and found an increase of the signal close to the magneto-structural transition temperature, which confirm that this technique is able to detect such a kind of transition when the sample surface is relatively clean and its roughness allows to detect the mentioned hyperfine interactions. We also see some additional THG anomaly at about $40 \mathrm{~K}$. We can suggest that this could be related to change of electronic structure of the compound when going below the magneto-structural transition due to enhanced interactions of the electronic subsystem with the critical bosons near the phase transformation. The observation of the temperature hysteresis in the THG signal can be related to the first order type of this transition. We have achieved SHG signal in both samples with signal increasing significantly below $80 \mathrm{~K}$ and reaching a maximum between 60 and $70 \mathrm{~K}$ (excepted for the second $\mathrm{Fe}_{1.08}$ Te sample studied for which only shoulder close to $70 \mathrm{~K}$ is found) and then the SHG signal decreased below about 40-50 K (excepted for the second $\mathrm{Fe}_{1.08}$ Te sample studied for which the SHG signal is maximal around $40 \mathrm{~K}$ and decreases only below 20-30 $\mathrm{K})$.

\section{References}

[1] K. Ishida, Y. Nakai, H. Hosono, J. Phys. Soc. Jpn. 78, 062001 (2009).

[2] D.C. Johnston, Adv. Phys. 59, 803 (2010). 
[3] R. Viennois, E. Giannini, D. van der Marel, R. Cerny, J. Solid State Chem. 183, 769 (2010).

[4] I.V. Kityk, J. Phys.: Cond. Mat. 6, 4119 (1994).

[5] K. Nouneh1, I.V. Kityk, R. Viennois, S. Benet, S. Charar, S. Paschen, K. Ozga, Phys. Rev. B 73, 035329 (2006).

[6] D.J. Hilton, R.P. Prasankumar, S.A. Trugman, A.J. Taylor, R.D. Averitt, J. Phys. Soc. Jpn. 75, 011006 (2006).

[7] N. Primozich, T.V. Shahbazyan, I.E. Perakis, D.S. Chemla, Phys. Rev. B 61, 2041 (2000).

[8] L. Stojchevska, P. Kusar, T. Mertelj, V.V. Kabanov, X. Lin, G.H. Cao, Z.A. Xu, D. Mihailovic, Phys. Rev. $B$ 82, 012505 (2010).
[9] K. Nouneh, R. Viennois, I.V. Kityk, F. Terki, S. Charar, S. Benet, S. Paschen, Phys. Stat. Sol. (b) 241, 3069 (2004).

[10] A. Bussmann-Holder, A.R. Bishop, Phys. Rev. B 70, 024104 (2004).

[11] E.E. Rodriguez, C. Stock, P. Zajdel, K.L. Krycka, C.F. Majkrzak, P. Zavalij, M.A. Green, Phys. Rev. B 84, 064403 (2011).

[12] I. Pallecchi, G. Lamura, M. Tropeano, M. Putti, R. Viennois, E. Giannini, D. Van der Marel, Phys. Rev. B 80, 214511 (2009). 